INPLASY

PROTOCOL

To cite: $\mathrm{Bi}$ et al. Comparison of magnetic resonance elastography and transient elastography in the diagnosis of hepatic fibrosis: a metaanalysis. Inplasy protocol 202160076. doi: 10.37766/inplasy2021.6.0076

Received: 22 June 2021

Published: 22 June 2021

Corresponding author: Junying Bi

bijunying1973@163.com

Author Affiliation: Hubei No. 3 People's Hospital of Jianghan University, Wuhan, China.

Support: None.

Review Stage at time of this submission: Data extraction.

Conflicts of interest:

None declared.

\section{Comparison of magnetic resonance elastography and transient elastography in the diagnosis of hepatic fibrosis: a meta-analysis}

\author{
Bi, J1; Liu, L2; Qin, T³.
}

Review question / Objective: To date, few studies have comprehensively compared the performance of magnetic resonance elastography (MRE) and transient elastography (TE) in the diagnosis of liver fibrosis. Therefore, we conducted a meta-analysis to evaluate and compare the diagnostic efficacy of these 2 techniques in patients with hepatic fibrosis in order to gain a better understanding of their overall diagnostic performance and aid in maximizing their clinical utility.

Condition being studied: Hepatic fibrosis. Systematic literature searches of the PubMed, EmBase, Cocharane Library, and China National Knowledge Infrastructure databases were carried out to identify studies that applied MRE and TE in the diagnosis of liver fibrosis. The combined sensitivity, specificity, positive and negative likelihood ratios, and diagnostic odds ratio (ORs) were estimated using a bivariate random effects model. Review Manager 5.2 was used to analyze the selected articles, and forest plot, sensitivity, and bias analyses were performed for the included literature. To determine the diagnostic efficacy of MRE and TE for liver fibrosis, pooled sensitivity and specificity analyses were conducted.

INPLASY registration number: This protocol was registered with the International Platform of Registered Systematic Review and Meta-Analysis Protocols (INPLASY) on 22 June 2021 and was last updated on 22 June 2021 (registration number INPLASY202160076).

\section{INTRODUCTION}

Review question / Objective: To date, few studies have comprehensively compared the performance of magnetic resonance elastography (MRE) and transient elastography (TE) in the diagnosis of liver fibrosis. Therefore, we conducted a metaanalysis to evaluate and compare the diagnostic efficacy of these 2 techniques in patients with hepatic fibrosis in order to gain a better understanding of their overall 
diagnostic performance and aid in maximizing their clinical utility.

Condition being studied: Hepatic fibrosis. Systematic literature searches of the PubMed, EmBase, Cocharane Library, and China National Knowledge Infrastructure databases were carried out to identify studies that applied MRE and TE in the diagnosis of liver fibrosis. The combined sensitivity, specificity, positive and negative likelihood ratios, and diagnostic odds ratio (ORs) were estimated using a bivariate random effects model. Review Manager 5.2 was used to analyze the selected articles, and forest plot, sensitivity, and bias analyses were performed for the included literature. To determine the diagnostic efficacy of MRE and TE for liver fibrosis, pooled sensitivity and specificity analyses were conducted.

\section{METHODS}

Participant or population: The 8 studies included in the meta-analysis involved a total of 847 participants (432 men and 415 women), with the study sample sizes ranging between 62 and 207.

Intervention: Magnetic resonance elastography.

\section{Comparator: Transient elastography.}

Study designs to be included: RCT or retrospective studies.

Eligibility criteria: (I) studies comparing MRE with TE for the diagnosis of hepatic fibrosis; (II) studies reporting the diagnostic sensitivity and specificity of the 2 imaging methods; and (III) studies comparing the parameters for each fibrosis stage.

Information sources: Systematic literature searches of the PubMed, EmBase, Cocharane Library, and China National Knowledge Infrastructure databases were carried out to identify studies that applied MRE and TE in the diagnosis of liver fibrosis.
Main outcome(s): MRE showed higher sensitivity than TE (OR $=0.62,95 \% \mathrm{Cl}$ : $0.41-$ 0.95, $P=0.03$ ). MRE also showed higher specificity than TE for diagnosing stage F2F4 liver fibrosis (OR $=0.41,95 \% \mathrm{Cl}$ : $0.27-$ $0.62, P<0.0001)$. There was no difference in the sensitivity of MRE and Te to F2-F4 hepatic fibrosis and the specificity of MRE and Te to F0-F1 hepatic fibrosis.

Quality assessment / Risk of bias analysis: The Cochrane risk of bias assessment tool was used to assess the included studies(Figure 2 and Figure 3). Overall Bbias was not an issue in any of the articles found in any article.

Strategy of data synthesis: All identified articles were included in the systematic review and examined using narrative synthesis.

Subgroup analysis: None.

Sensitivity analysis: The heterogeneity may be attributed to the differences in the results of the studies. After the exclusion of Tafur's 2020 study, 12 changed from $0 \%$ to $14 \%$, and the $P$ value changed from 0.03 to 0.04 (Figure 8), indicating the reliability of the results of this article.

Country(ies) involved: China.

Keywords: Magnetic resonance elastography (MRE); transient elastography (TE); hepatic fibrosis; liver fibrosis; metaanalysis.

Contributions of each author:

Author 1 - Junying Bi.

Author 2 - Liangjin Liu.

Author 3 - Tao Qin. 